\title{
Closures and Interiors Redefined, and Some Types of Compactness in Ordinary Smooth Topological Spaces
}

\author{
Jeong Gon Lee, Pyung Ki Lim and Kul Hur ${ }^{\dagger}$ \\ Division of Mathematics and Informational Statistics, and Nanoscale Science and Technology Institute, \\ Wonkwang University
}

\begin{abstract}
We give a new definition of ordinary smooth closure and ordinary smooth interior of an ordinary subset in an ordinary smooth topological space which have almost all the properties of the corresponding operators in a classical topological space. As a consequence of these definitions we reduce the additional hypotheses in the results of [1] and also generalize several properties of the types of compactness in [1].
\end{abstract}

Key Words: Ordinary Smooth Topological Space, Ordinary Smooth Closure [resp. Interior], Ordinary Smooth [resp. Almost and Near] Compacteness.

\section{Introduction}

In 1985, Šostak [2] introduced the concept of a fuzzy topology on a set $X$ as a mapping $\tau: I^{X} \rightarrow I$ satisfying some natural axioms, where $I=[0,1]$ and $I^{X}$ denotes the set of all fuzzy sets in $X$. In 1992, Chattopadyay et al. [3] investigated the same structures. They called the mapping $\tau: I^{X} \rightarrow I$ as a "gradation of openness of fuzzy sets in $X "$. In the same year, Ramadan [4] proposed a similar definition of a fuzzy topology in Sostak's sense called "smooth topology" replacing $I$ by possibly more

접수일자 : 2012년 9월 27일

심사(수정)일자 : 2012년 12월 11일

게재확정일자 : 2012년 12월 18일

${ }^{\dagger}$ Corresponding author

This is an Open-Access article distributed under the terms of the Creative Commons Attribution Non-Commercial License(http://creativecommons.org/licenses/by-nc/3.0) which permits unrestricted non-commercial use, distribution, and reproduction in any medium, provided the original work is properly cited. general lattices. In particular, Kim et al. [5] and M. ElDardery et al. [6] studied smooth neighborhood structures and smooth uniform spaces, respectively. Also, Kim [7] investigated separation axioms in smooth fuzzy topological spaces.

On the other hand, in 1991, Ying [8] gave the definition of fuzzifying topology on $X$ considering a "degree of openness of ordinary subsets of $X$ ". Recently, Hur et al. [9] gave the similar definition called an ordinary smooth topolgy on $X$ as a mapping $\tau: 2^{X} \rightarrow I$ satisfying three axioms, where 2 denotes the two points set $\{0,1\}$. In particular, Chae et al. [10] studied the set $\operatorname{OST}(\mathrm{X})$ of all ordinary smooth topologies on $X$ in the sense of a lattice. Moreover, Lim et al. [1] introduced and investigated closures, interiors and the types of compactness in ordinary smooth topological spaces. However the results obtained include additional conditions since the ordinary smooth closure and ordinary smooth interior defined there do not have such nice properties as the closure and interior operators in a classical topological space.

In this paper, we give a new definition of ordinary smooth closure and ordinary smooth interior of an ordinary subset in an ordinary smooth topological space which 
have almost all the properties of the corresponding operators in a classical topological space. As a consequence of these definitions we reduce the additional hypotheses in the results of [1] and also generalize several properties of the types of compactness in [1].

\section{Preliminaries}

Let $2=\{0,1\}$ and let $2^{X}$ denote the set of all ordinary subsets of a set $X$.

Definition 2.1[9] Let $X$ be a nonempty set. Then a mapping $\tau: 2^{X} \rightarrow I$ is called an ordinary smooth topology (in short, ost) on $X$ or a gradation of openness of ordinary subsets of $X$ if $\tau$ satisfies the following axioms:

$\left(\mathrm{OST}_{1}\right) \tau(\emptyset)=\tau(X)=1$.

$\left(\mathrm{OST}_{2}\right) \tau(A \cap B) \geq \tau(A) \wedge \tau(B), \forall A, B \in 2^{X}$.

$\left(\mathrm{OST}_{3}\right) \tau\left(\bigcup_{\alpha \in \Gamma} A_{\alpha}\right) \geq \bigwedge_{\alpha \in \Gamma} \tau\left(A_{\alpha}\right), \forall\left\{A_{\alpha}\right\} \subset 2^{X}$.

The pair $(X, \tau)$ is called an ordinary smooth topological space (in short, osts).

We will denote the set of all osts's on $X$ as $\operatorname{OST}(\mathrm{X})$.

Remark 2.2 Ying [8] called the mapping $\tau: 2^{X} \rightarrow I$ [resp. $\tau: I^{X} \rightarrow 2$ and $\left.\tau: I^{X} \rightarrow I\right]$ satisfying the axioms in Definition 2.1 as a fuzzyfying topology [resp. fuzzy topology and bifuzzy topology] on $X$.

Remark 2.3 If $I=2$, then Definition 2.1 coincides with the known definition of the classical topology.

Definition 2.4[9] Let $X$ be a nonempty set. Then a mapping $\mathcal{C}: 2^{X} \rightarrow I$ is called an ordinary smooth cotopology (in short, osct) on $X$ or a gradation of closedness of ordinary subsets of $X$ if $\mathcal{C}$ satisfies the following axioms :

$$
\begin{aligned}
& \left(\mathrm{OSCT}_{1}\right) \mathcal{C}(\emptyset)=\mathcal{C}(X)=1 . \\
& \left(\mathrm{OSCT}_{2}\right) \mathcal{C}(A \cup B) \geq \mathcal{C}(A) \wedge \mathcal{C}(B), \forall A, B \in 2^{X} . \\
& \left(\mathrm{OSCT}_{3}\right) \mathcal{C}\left(\bigcap_{\alpha \in \Gamma} A_{\alpha}\right) \geq \bigwedge_{\alpha \in \Gamma} \mathcal{C}\left(A_{\alpha}\right), \forall\left\{A_{\alpha}\right\} \subset 2^{X} .
\end{aligned}
$$

The pair $(X, \mathcal{C})$ is called an ordinary smooth cotopological space (in short, oscts).

We will denote the set of all oscts's on $X$ as $\operatorname{OSCT}(X)$.

Remark 2.5 If $I=2$, then Definition 2.4 also coincides with the known definition of the classical topology.

Result 2.A[9, Proposition 2.7] Let $X$ be a nonempty set. We define two mappings $f: \operatorname{OST}(\mathrm{X}) \rightarrow \mathrm{OSCT}(\mathrm{X})$ and $g: \operatorname{OSCT}(\mathrm{X}) \rightarrow \operatorname{OST}(\mathrm{X})$ as follows, respectively :

$$
[f(\tau)](A)=\tau\left(A^{c}\right), \forall \tau \in \mathrm{OST}(X), \forall A \in 2^{X}
$$

and

$$
[g(\mathcal{C})](A)=\mathcal{C}\left(A^{c}\right), \forall \mathcal{C} \in \operatorname{OSCT}(X), \forall A \in 2^{X} .
$$

Then $f$ and $g$ are well-defined. Furthermore $g \circ f=$ $i d_{\mathrm{OST}(\mathrm{X})}$ and $f \circ g=i d_{\mathrm{OSCT}(\mathrm{X})}$.

Remark 2.6 Let $f(\tau)=\mathcal{C}_{\tau}$ and $g(\mathcal{C})=\tau_{\mathcal{C}}$. Then, Result 2.A, we can easily see that $\tau_{\mathcal{C}_{\tau}}=\tau$ and $\mathcal{C}_{\tau_{\mathcal{C}}}=\mathcal{C}$.

Definition 2.7[9] Let $(X, \tau)$ be an osts and let $r \in I$. Then we define two ordinary subsets of $X$ as follows :

$$
[\tau]_{r}=\left\{A \in 2^{X}: \tau(A) \geq r\right\}
$$

and

$$
[\tau]_{r}^{*}=\left\{A \in 2^{X}: \tau(A)>r\right\} .
$$

We call these the $r$-level set and the strong $r$-level set of $\tau$, respectively.

It is clear that $[\tau]_{0}=2^{X}$, the classical discrete topology on $X$ and $[\tau]_{1}^{*}=\emptyset$. Also it can be easily seen that $[\tau]_{r}^{*} \subset[\tau]_{r}$ for each $r \in I$.

Result 2.B[9, Proposition 2.10] Let $(X, \tau)$ be an osts and let $T(X)$ denote the set of all classical topologies on $X$. Then :

(a) $[\tau]_{r} \in \mathrm{T}(X), \forall r \in I$.

(a) $)^{\prime}[\tau]_{r}^{*} \in \mathrm{T}(X), \forall r \in I_{1}$.

(b) For any $r, s \in I$, if $r \leq s$, then $[\tau]_{s} \subset[\tau]_{r}$ and $[\tau]_{s}^{*} \subset$ $[\tau]_{r}^{*}$.

(c) $[\tau]_{r}=\bigcap_{s<r}[\tau]_{s}, \forall r \in I_{0}$.

(c) ${ }^{\prime}[\tau]_{r}^{*}=\bigcup_{s>r}[\tau]_{s}^{*}, \forall r \in I_{1}$, where $I_{1}=[0,1)$ and $I_{0}=$ $(0,1]$.

Definition 2.8[1] Let $(X, \tau)$ be an osts, let $r \in I$ and let $A \in 2^{X}$. Then the $[\tau]_{r}$ - closure [resp. $[\tau]_{r}$-interior] of 
$A$, denoted by $\operatorname{cl}_{r}(A)$ or $\bar{A}_{r}$ [resp. $\operatorname{int}_{r}(A)$ or $\left.\stackrel{\circ}{A_{r}}\right]$, is defined by

$$
\operatorname{cl}_{r}(A) \text { or } \bar{A}_{r}=\bigcap\left\{F \in 2^{X}: F \in F_{[\tau]_{r}} \text { and } A \subset F\right\}
$$

$\left[\operatorname{resp}, \operatorname{int}_{r}(A)\right.$ or $\stackrel{\circ}{A}_{r}=\bigcup\left\{U \in 2^{X}: U \in[\tau]_{r}\right.$ and $\left.\left.U \subset A\right\}\right]$, where $F_{[\tau]_{r}}$ denotes the set of all $[\tau]_{r^{-}}$closed sets in $X$.

\section{Ordinary smooth closure and interior} redefined

Definition 3.1 Let $(X, \tau)$ be an osts and let $A \in 2^{X}$. Then the ordinary smooth closure [resp. ordinary smooth interior] of $A$ in $X$, denoted by $\bar{A}$ [resp. $\stackrel{\circ}{A}$ ], is defined by

$$
\begin{gathered}
\bar{A}=\bigcap\left\{F \in 2^{X}: A \subset F \text { and } \mathcal{C}_{\tau}(\mathrm{F})>0\right\} \\
\text { [resp. } \left.\stackrel{\circ}{\mathrm{A}}=\bigcup\left\{\mathrm{U} \in 2^{\mathrm{X}}: \mathrm{U} \subset \mathrm{A} \text { and } \tau(\mathrm{U})>0\right\}\right] .
\end{gathered}
$$

Proposition 3.2 Let $(X, \tau)$ be an ordinary smooth topological space and let $A, B \in 2^{X}$. Then:
(a) If $A \subset B$, then $\stackrel{\circ}{A} \subset \stackrel{\circ}{B}$ and $\bar{A} \subset \bar{B}$.
(b) $(\stackrel{\circ}{A})^{c}=\overline{\left(A^{c}\right)}$.
(c) $\stackrel{\circ}{A}=\left(\overline{\left(A^{c}\right)}\right)^{c}$.
(d) $\bar{A}=\left(\left(A^{c}\right)^{\circ}\right)^{c}$.
(e) $(\bar{A})^{c}=\left(A^{c}\right)^{\circ}$.

Proof. (a) It follows directly from Definition 3.1.

(b)

$$
\begin{aligned}
(\stackrel{\circ}{A})^{c}= & \left(\bigcup\left\{U \in 2^{X}: U \subset A \text { and } \tau(\mathrm{U})>0\right\}\right)^{\mathrm{c}} \\
= & \bigcap\left\{U^{c} \in 2^{X}: A^{c} \subset U^{c} \text { and } \tau(\mathrm{U})\right. \\
& \left.=\mathcal{C}_{\tau}\left(U^{c}\right)>0\right\} \\
= & \bigcap\left\{F \in 2^{X}: A^{c} \subset F \text { and } \mathcal{C}_{\tau}(\mathrm{F})>0\right\} \\
= & \overline{\left(A^{c}\right)} .
\end{aligned}
$$

(c), (d) and (e) can be easily derived from (b).

Remark 3.3 Let $(X, T)$ be a classical topological space. We define a mapping $\tau_{T}: 2^{X} \rightarrow I$ as follows: For each $A \in 2^{X}$,

$$
\tau_{T}(A)= \begin{cases}1, & \text { if } \quad \mathrm{A} \in \mathrm{T}, \\ 0, & \text { if } \quad \mathrm{A} \notin \mathrm{T} .\end{cases}
$$

Then we can easily see that $\tau_{T} \in \operatorname{OST}(\mathrm{X})$. Moreover, we can identify $\tau_{T}$ with $T$, and we can also identify $\mathcal{C}_{\tau_{T}}$ with $T$. Thus, for each $A \in 2^{X}$, the $\tau_{T}$-ordinary smooth closure and $\tau_{T}$-ordinary interior of $A$ are followings, respectively:

$$
\begin{aligned}
\bar{A} & =\bigcap\left\{F \in 2^{X}: A \subset F \quad \text { and } \quad \mathcal{C}_{\tau_{\mathrm{T}}}(\mathrm{F})>0\right\} \\
& =\bigcap\left\{F \in 2^{X}: A \subset F \quad \text { and } \quad \mathrm{F}^{\mathrm{c}} \in \mathrm{T}\right\}
\end{aligned}
$$

and

$$
\begin{aligned}
\stackrel{\circ}{A} & =\bigcup\left\{U \in 2^{X}: U \subset A \text { and } \quad \tau(\mathrm{U})>0\right\} \\
& =\bigcup\left\{U \in 2^{X}: U \subset A \text { and } \quad \mathrm{U} \in \mathrm{T}\right\} .
\end{aligned}
$$

So this shows that $\bar{A}$ and $\stackrel{\circ}{A}$ are exactly closure and interior of $A$ in a classical topological space $(X, \tau)$, respectively.

Proposition 3.4 Let $(X, \tau)$ be an osts and let $A, B \in 2^{X}$. Then:
(a) $\stackrel{\circ}{X}=X$.
(b) $\stackrel{\circ}{A} \subset A$.
(c) $(\stackrel{\circ}{A})^{\circ}=\stackrel{\circ}{A}$.
(d) $(A \cap B)^{\circ} \subset \stackrel{\circ}{A} \cap \stackrel{\circ}{B}$.

Proof. From Definition 3.1, (a) and (b) can be easily obtained.

(c) By (b), it is clear that $(\stackrel{\circ}{A})^{\circ} \subset \stackrel{\circ}{A}$. On the other hand,

$$
\begin{aligned}
(\stackrel{\circ}{A})^{\circ}= & \bigcup\left\{U \in 2^{X}: U \subset \stackrel{\circ}{A} \text { and } \tau(\mathrm{U})>0\right\} \\
= & \bigcup\left\{U \in 2^{X}: \tau(U)>0\right. \text { and } \\
& \left.U \subset \bigcup\left\{V \in 2^{X}: V \subset A \text { and } \tau(\mathrm{V})>0\right\}\right\} \\
\supset & \bigcup \stackrel{\circ}{\bigcup}\left\{U \in 2^{X}: U \subset A \text { and } \mathrm{U} \subset \mathrm{A}\right\} \\
= & \stackrel{ }{A} .
\end{aligned}
$$

So $(\stackrel{\circ}{A})^{\circ}=\stackrel{\circ}{A}$.

(d) It is obvious that $A \cap B \subset A$ and $A \cap B \subset B$. Then, by (b), $(A \cap B)^{\circ} \subset \stackrel{\circ}{A}$ and $(A \cap B)^{\circ} \subset \stackrel{\circ}{B}$. Thus $(A \cap B)^{\circ} \subset \stackrel{\circ}{A} \cap \stackrel{\circ}{B}$.

Proposition 3.5 Let $(X, \tau)$ be an ordinary smooth topological space and let $A, B \in 2^{X}$. Then:
(a) $\bar{\emptyset}=\emptyset$.
(b) $A \subset \bar{A}$.
(c) $\overline{(\bar{A})}=\bar{A}$.
(d) $\bar{A} \cup \bar{B} \subset \overline{A \cup B}$. 
Proof. The proofs are similar to that of Proposition 3.5.

Proposition 3.6 Let $(X, \tau)$ be an osts and let $A \in 2^{X}$.

Then:

(a) If $\tau(A)>0$, then $A=\stackrel{\circ}{A}$.

(b) If $\mathcal{C}_{\tau}(A)>0$, then $A=\bar{A}$.

(c) If there is an $r \in I_{0}$ such that $A=\overline{A_{r}}$, then $A=\bar{A}$.

(d) If there is an $r \in I_{0}$ such that $A=\stackrel{\circ}{A}$, then $A=\stackrel{\circ}{A}$.

Proof. (a) Suppose $\tau(A)>0$. Then $A \in\left\{U \in 2^{X}\right.$ : $U \subset A$ and $\tau(\mathrm{U})>0\}$. Thus $A \subset \bigcup\left\{U \in 2^{X}\right.$ : $U \subset A$ and $\tau(\mathrm{U})>0\}$. By Definition 3.1, $A \subset \stackrel{\circ}{A}$. By Proposition 3.5 (b), clearly $\stackrel{\circ}{A} \subset A$. So $A=\stackrel{\circ}{A}$.

(b) Suppose $\mathcal{C}_{\tau}(A)>0$. Then $\tau\left(A^{c}\right)=\mathcal{C}_{\tau}(A)>0$. Thus, by (a), $A^{c}=\left(A^{c}\right)^{\circ}$. So $A=\left(\left(A^{c}\right)^{\circ}\right)^{c}$. Hence, by Proposition 3.2 (d), $A=\bar{A}$.

(c) Suppose the sufficient condition holds. Then

$$
\begin{aligned}
\bar{A} & =\bigcap\left\{F \in 2^{X}: A \subset F \quad \text { and } \quad \mathcal{C}_{\tau}(\mathrm{F})>0\right\} \\
& =\bigcap\left[\bigcap_{s \in I_{0}}\left\{F \in 2^{X}: A \subset F \quad \text { and } \quad \mathcal{C}_{\tau}(\mathrm{F}) \geq \mathrm{s}\right\}\right] \\
& =\bigcap_{s \in I_{0}}\left[\bigcap\left\{F \in 2^{X}: A \subset F \quad \text { and } \quad \mathrm{F} \in\left[\mathcal{C}_{\tau}\right]_{\mathrm{s}}\right\}\right] \\
& =\bigcap_{s \in I_{0}} \overline{A_{s}} .
\end{aligned}
$$

Thus

$$
\begin{aligned}
A & \subset \bar{A} \quad[\text { By Proposition } 3.6(\mathrm{~b})] \\
& =\bigcap_{s \in I_{0}} \bar{A}_{s} \\
& \subset \overline{A_{r}} \\
& =A . \quad[\text { By the hypothesis }] .
\end{aligned}
$$

So $\bar{A}=A$.

(d) Suppose the sufficient condition holds. Then

$$
\begin{aligned}
\stackrel{\circ}{A} & =\bigcup\left\{U \in 2^{X}: U \subset A \text { and } \tau(\mathrm{U})>0\right\} \\
& =\bigcup\left\{U \in 2^{X}: \tau(U)>0 \text { and } \quad \mathrm{U} \subset \stackrel{\circ}{\mathrm{r}}_{\mathrm{r}}\right\}
\end{aligned}
$$

[By the hypothesis]

$$
\begin{aligned}
=\bigcup[ & \left\{U \in 2^{X}: \tau(U)>0\right. \text { and } \\
& \left.U \subset \bigcup\left\{V \in 2^{X}: \tau(V) \geq r \text { and } \mathrm{V} \subset \mathrm{A}\right\}\right] .
\end{aligned}
$$

On the other hand,

$$
\begin{aligned}
\tau\left(\stackrel{\circ}{A}_{r}\right) & \geq \bigwedge\left\{\tau(V): V \in 2^{X}, \tau(V) \geq r \quad \text { and } \quad \mathrm{U} \subset \mathrm{A}\right\} \\
& \geq r>0 .
\end{aligned}
$$

Thus $\underset{\circ}{\AA_{r}} \in\left\{U \in 2^{X}: \tau(U)>0\right.$ and $\left.\mathrm{U} \subset \stackrel{\circ}{\mathrm{A}}_{\mathrm{r}}\right\}$. So $\stackrel{\circ}{A}_{r} \subset \stackrel{\circ}{A}$. By Proposition 3.5 (b) and the hypothesis,

$$
A=\stackrel{\circ}{A} \subset \stackrel{\circ}{A} \subset A .
$$

Hence $\stackrel{\circ}{A}=A$. This completes the proof.

Definition 3.7 Let $\left(X, \tau_{1}\right)$ and $\left(Y, \tau_{2}\right)$ be two osts's. Then a mapping $f: X \rightarrow Y$ is said to be :

(i)[8] ordinary smooth continuous if $\tau_{2}(A) \leq$ $\tau_{1}\left(f^{-1}(A)\right), \forall A \in 2^{Y}$.

(ii)[4] ordinary weakly smooth continuous if for each $A \in 2^{Y}$,

$$
\tau_{2}(A)>0 \Rightarrow \tau\left(f^{-1}(A)\right)>0
$$

It is clear that if $f$ is ordinary smooth continuous, then $f$ is ordinary weakly smooth continuous.

Proposition 3.8 Let $\left(X, \tau_{1}\right)$ and $\left(Y, \tau_{2}\right)$ be two osts's and let a mapping $f: X \rightarrow Y$ be ordinary weakly smooth continuous. Then:

(a) $f(\bar{A}) \subset \overline{f(A)}, \quad \forall A \in 2^{X}$.

(b) $\overline{f^{-1}(B)} \subset f^{-1}(\bar{B}), \quad \forall B \in 2^{Y}$.

(c) $f^{-1}(\stackrel{\circ}{B}) \subset\left(f^{-1}(B)\right)^{\circ}, \quad \forall B \in 2^{Y}$.

Proof. (a) Let $A \in 2^{X}$. Then

$$
\begin{aligned}
& f^{-1} \overline{(f(A))} \\
& =f^{-1}\left(\bigcap\left\{F \in 2^{Y}: \mathcal{C}_{\tau_{2}}(F)>0 \text { and } f(A) \subset F\right\}\right) \\
& =\bigcap\left\{f^{-1}(F) \in 2^{X}: F \in 2^{Y}, \mathcal{C}_{\tau_{2}}(F)>0\right. \\
& \left.\quad \text { and } A \subset f^{-1}(F)\right\} \\
& \supset \cap\left\{f^{-1}(F) \in 2^{X}: F \in 2^{Y}, \mathcal{C}_{\tau_{1}}\left(f^{-1}(F)\right)>0\right. \\
& \left.\quad \text { and } A \subset f^{-1}(F)\right\}
\end{aligned}
$$

[Since $f$ is ordinary weakly smooth continuous]

$$
=\bigcap\left\{B \in 2^{X}: \mathcal{C}_{\tau_{1}}(B)>0 \text { and } A \subset B\right\}
$$$$
=\bar{A} \text {. }
$$

Thus $f(\bar{A}) \subset \overline{f(A)}$.

(b) Let $B \in 2^{Y}$. Then $f^{-1}(B) \in 2^{X}$.

$$
\begin{aligned}
f \overline{\left(f^{-1}(B)\right)} & \subset \overline{f\left(f^{-1}(B)\right)} \quad[\text { By (a) }] \\
& =\bar{B}
\end{aligned}
$$

So

$$
\overline{f^{-1}(B)} \subset f^{-1}\left[f\left(\overline{f^{-1}(B)}\right)\right] \subset f^{-1}(\bar{B}) .
$$


Hence $\overline{f^{-1}(B)} \subset f^{-1}(\bar{B})$.

(c) Let $B \in 2^{Y}$. Then

$$
\begin{array}{rlr}
f^{-1}\left(\overline{B^{c}}\right) & \left.=f^{-1}(\stackrel{\circ}{B})^{c}\right) & {[\text { By Proposition 3.2 (b) }]} \\
& =\left(f^{-1}(\stackrel{\circ}{B})\right)^{c} & \\
& \supset \overline{f^{-1}\left(B^{c}\right)} & {[\text { By (b) and Proposition 3.2 (c) }]} \\
& =\overline{\left(f^{-1}(B)\right)^{c}} \\
& =\left[\left(f^{-1}(B)\right)^{\circ}\right]^{c} & {[\text { By Proposition 3.2 (b) }] .}
\end{array}
$$

So $f^{-1}(\stackrel{\circ}{B}) \subset\left(f^{-1}(B)\right)^{\circ}$.

The following is the immediate result of Definition 3.7 and Proposition 3.8.

Corollary 3.9 Let $\left(X, \tau_{1}\right)$ and $\left(Y, \tau_{2}\right)$ be two osts's and let $f: X \rightarrow Y$ be ordinary smooth continuous. Then:
(a) $f(\bar{A}) \subset \overline{f(A)}, \quad \forall A \in 2^{X}$.
(b) $\overline{f^{-1}(B)} \subset f^{-1}(\bar{B}), \quad \forall B \in 2^{Y}$.
(c) $f^{-1}(\stackrel{\circ}{B}) \subset\left(f^{-1}(B)\right)^{\circ}, \quad \forall B \in 2^{Y}$.

Definition 3.10[11] Let $\left(X, \tau_{1}\right)$ and $\left(Y, \tau_{2}\right)$ be two ordinary smooth topological spaces. Then a mapping $f: X \rightarrow Y$ is said to be:

(i)ordinary smooth open if $\tau_{1}(A) \leq \tau_{2}(f(A))$, $\forall A \in 2^{X}$.

(ii) ordinary smooth closed if $\mathcal{C}_{\tau_{1}}(A) \leq \mathcal{C}_{\tau_{2}}(f(A))$, $\forall A \in 2^{X}$.

Proposition 3.11 Let $\left(X, \tau_{1}\right)$ and $\left(Y, \tau_{2}\right)$ be two ordinary smooth topological spaces. Suppose $f: X \rightarrow Y$ is ordinary smooth open, then $f(\stackrel{\circ}{A}) \subset(f(A))^{\circ}$ for each $A \in 2^{X}$.

Proof. Let $A \in 2^{X}$. Then

$$
\begin{aligned}
f(\stackrel{\circ}{A})= & f\left(\bigcup\left\{U \in 2^{X}: \tau_{1}(U)>0 \text { and } U \subset A\right\}\right) \\
\subset & \bigcup\left\{f(U) \in 2^{Y}: U \in 2^{X}, \tau_{1}(U)>0\right. \\
& \text { and } f(U) \subset f(A)\} \\
\subset & \bigcup\left\{f(U) \in 2^{Y}: U \in 2^{X}, \tau_{2}(f(U))>0\right. \\
& \text { and } f(U) \subset f(A)\} \\
& \quad[\text { Since } f \text { is ordinary smooth open }] \\
= & \bigcup\left\{V \in 2^{Y}: \tau_{2}(V)>0 \text { and } V \subset f(A)\right\} \\
= & (f(A))^{\circ} .
\end{aligned}
$$

Thus $f(\stackrel{\circ}{A}) \subset(f(A))^{\circ}$.

\section{Some types of ordinary smooth compactness}

For an ordinary smooth topological space $(X, \tau)$, we will denote the set $\left\{A \in 2^{X}: \tau(A)>0\right\}$ as $S(\tau)$ and we will call it as the support of $\tau$.

Definition 4.1[1] An ordinary smooth topological space $(X, \tau)$ is said to be:

(i) ordinary smooth compact if for every family $\left\{A_{\alpha}\right\}_{\alpha \in \Gamma}$ in $S(\tau)$ covering $X$, these is a finite subset $\Gamma_{0}$ of $\Gamma$ such that $\bigcup_{\alpha \in \Gamma_{0}} A_{\alpha}=X$.

(ii) ordinary smooth nearly compact if for every family $\left\{A_{\alpha}\right\}_{\alpha \in \Gamma}$ in $S(\tau)$ covering $X$, there is a finite subset $\Gamma_{0}$ of $\Gamma$ such that $\bigcup_{\alpha \in \Gamma_{0}}\left(\overline{A_{\alpha}}\right)^{\circ}=X$.

(iii) ordinary smooth almost compact if for every family $\left\{A_{\alpha}\right\}_{\alpha \in \Gamma}$ in $S(\tau)$ covering $X$, there is a finite subset $\Gamma_{0}$ of $\Gamma$ such that $\bigcup_{\alpha \in \Gamma_{0}} \overline{A_{\alpha}}=X$.

Proposition 4.2 Ordinary smooth compactness $\Rightarrow$ ordinary smooth near compactness $\Rightarrow$ ordinary smooth almost compactness.

Proof. Suppose $(X, \tau)$ be an ordinary smooth compact space. Let $\left\{A_{\alpha}\right\}_{\alpha \in \Gamma}$ be any family in $S(\tau)$ covering $X$, i.e., $\bigcup_{\alpha \in \Gamma} A_{\alpha}=X$. Then, by the hypothesis, $\exists$ a finite subset $\Gamma_{0}$ of $\Gamma$ such that $\bigcup_{\alpha \in \Gamma_{0}} A_{\alpha}=X$. Since $\left\{A_{\alpha}\right\}_{\alpha \in \Gamma}$ is a family in $S(\tau), A_{\alpha} \in S(\tau)$ for each $\alpha \in \Gamma$, i.e., $\tau\left(A_{\alpha}\right)>0$ for each $\alpha \in \Gamma$. Thus, by Proposition 3.6 (a), $A_{\alpha}=\stackrel{\circ}{A}$ for each $\alpha \in \Gamma$. So

$$
A_{\alpha}=\stackrel{\circ}{A}_{\alpha} \subset\left(\overline{A_{\alpha}}\right)^{\circ} \quad \text { for each } \alpha \in \Gamma .
$$

Hence

$$
X=\bigcup_{\alpha \in \Gamma_{0}} A_{\alpha} \subset \bigcup_{\alpha \in \Gamma_{0}}\left(\overline{A_{\alpha}}\right)^{\circ}, \quad \text { i.e., } \bigcup_{\alpha \in \Gamma_{0}}\left(\overline{A_{\alpha}}\right)^{\circ}=X .
$$

Therefore $(X, \tau)$ is ordinary smooth nearly compact.

Now suppose $(X, \tau)$ is ordinary smooth nearly compact. Let $\left\{A_{\alpha}\right\}_{\alpha \in \Gamma}$ be any family in $S(\tau)$ covering $X$. 
Then, by the hypothesis, $\exists$ a finite subset $\Gamma_{0}$ of $\Gamma$ such that $\bigcup_{\alpha \in \Gamma_{0}}\left(\overline{A_{\alpha}}\right)^{\circ}=X$. On the other hand,

$$
A_{\alpha}=\stackrel{\circ}{A_{\alpha}}
$$

[Since $\left\{A_{\alpha}\right\}_{\alpha \in \Gamma}$ is a family in $S(\tau)$, by Proposition 3.6 (a)]

$$
\begin{aligned}
& \subset\left(\overline{A_{\alpha}}\right)^{\circ} \\
& \subset \overline{A_{\alpha}} \text { for each } \alpha \in \Gamma \quad[\text { By Proposition } 3.5(\mathrm{~b})] .
\end{aligned}
$$

Thus $X=\bigcup_{\alpha \in \Gamma_{0}}\left(\overline{A_{\alpha}}\right)^{\circ} \subset \bigcup_{\alpha \in \Gamma_{0}} \overline{A_{\alpha}}$, i.e., $\quad \mathrm{X}=$ $\bigcup_{\alpha \in \Gamma_{0}} \overline{\mathrm{A}_{\alpha}}$. Hence $(X, \tau)$ is ordinary smooth almost compact.

In a classical topological space, the converses of these two implications are not valid for compactness, near compactness and almost compactness. Thus, the converse implications in Proposition 4.2 are not true, in general.

Proposition 4.3 Let $\left(X, \tau_{1}\right)$ and $\left(Y, \tau_{2}\right)$ be two osts's and let $f: X \rightarrow Y$ be surjective and ordinary weakly smooth continuous. If $\left(X, \tau_{1}\right)$ is ordinary smooth almost compact, then so is $\left(Y, \tau_{2}\right)$.

Proof. Suppose $f: X \rightarrow Y$ is surjective and ordinary weakly smooth continuous. Let $\left\{A_{\alpha}\right\}_{\alpha \in \Gamma}$ be any family in $S\left(\tau_{2}\right)$ covering $Y$, i.e., $\bigcup_{\alpha \in \Gamma} A_{\alpha}=Y$. Then $f^{-1}(Y)=X=\bigcup_{\alpha \in \Gamma} f^{-1}\left(A_{\alpha}\right)$. Since $f$ is ordinary weakly smooth continuous, $\left\{f^{-1}\left(A_{\alpha}\right)\right\}_{\alpha \in \Gamma}$ is a family in $S\left(\tau_{1}\right)$. Sice $\left(X, \tau_{1}\right)$ is ordinary smooth almost compact, $\exists$ a finite subset $\Gamma_{0}$ of $\Gamma$ such that $X=\bigcup_{\alpha \in \Gamma_{0}} \overline{f^{-1}\left(A_{\alpha}\right)}$. Thus

$$
\begin{aligned}
Y & =f(X) \quad[\text { Since } f \text { is surjective }] \\
& =f\left(\bigcup_{\alpha \in \Gamma_{0}} \overline{f^{-1}\left(A_{\alpha}\right)}\right) \\
& =\bigcup_{\alpha \in \Gamma_{0}} f\left(\overline{\left(f^{-1}\left(A_{\alpha}\right)\right)} \quad \quad\right. \text { [By Proposition 3.8 (a)] } \\
& \subset \bigcup_{\alpha \in \Gamma_{0}} \overline{f\left(f^{-1}\left(A_{\alpha}\right)\right)} \\
& =\bigcup_{\alpha \in \Gamma_{0}} \overline{A_{\alpha}} \quad[\text { Since } f \text { is surjective }]
\end{aligned}
$$

So $Y=\bigcup_{\alpha \in \Gamma_{0}} \overline{A_{\alpha}}$. Hence $\left(Y, \tau_{2}\right)$ is ordinary smooth almost compact.
The following is the immediate result of propositions 4.2 and 4.3 .

Corollary 4.4 Let $\left(X, \tau_{1}\right)$ and $\left(Y, \tau_{2}\right)$ be two osts's and let $f: X \rightarrow Y$ be surjective and ordinary weakly smooth continuous. If $\left(X, \tau_{1}\right)$ is ordinary smooth nearly compact, then $\left(Y, \tau_{2}\right)$ is ordinary smooth almost compact.

The following is the immediate result of Definition 3.7, Proposition 4.3 and Corollary 4.4.

Corollary 4.5 Let $\left(X, \tau_{1}\right)$ and $\left(Y, \tau_{2}\right)$ be two osts's and let $f: X \rightarrow Y$ be surjective and ordinary smooth continuous.

(a) If $\left(X, \tau_{1}\right)$ is ordinary smooth almost compact, then so is $\left(Y, \tau_{2}\right)$.

(b) If $\left(X, \tau_{1}\right)$ is ordinary smooth nearly compact, then $\left(Y, \tau_{2}\right)$ is ordinary smooth almost compact.

Definition 4.6[1] An osts $(X, \tau)$ is said to be ordinary smooth regular if $A=\cup\left\{F \in 2^{X}: \tau(F) \geq\right.$ $\tau(A)$ and $\overline{\mathrm{F}} \subset \mathrm{A}\}$ for each $A \in S(\tau)$.

Result 4.A[1, Proposition 5.4(a)] Every ordinary smooth almost compact and ordinary smooth regular space is ordinary smooth compact.

The following is the immediate result of Proposition 4.2 and Result 4.A.

Proposition 4.7 Every ordinary smooth nearly compact and ordinary smooth regular space is ordinary smooth compact.

\section{References}

[1] J. G. Lee, K. Hur and P. K. Lim, Closure, Interior and Compactness in Ordinary Smooth Topological Spaces, To be submitted. 
[2] A. P. Šostak, On a Fuzzy Topological Structure, Rend. Circ. Matem. Palermo (2) Suppl. 11(1985), 89-103.

[3] K. C. Chattopadhyay, R. N. Haza and S. K. Samanta, Gradation of openness : Fuzzy Topology, Fuzzy Sets and Systems 49(1992), 237-242.

[4] A. A. Ramadan, Smooth topological spaces, Fuzzy Sets and Systems 48(1992), 371-375.

[5] Y. C. Kim, M. K. El-Gayyar and A. A. Ramadan, Smooth Neighberhood Structures, Korean Institute of Intelligent Systems 12(2)(2002), 187-191.

[6] M. El-Dardery, Y. C. Kim and A. A. Ramadan, Smooth Uniform Spaces, IJFIS. 2(1)(2002), 90-95.

[7] Y. C. Kim, Separation Axioms in Smooth Fuzzy Topological Spaces, Korean Institute of Intelligent Systems 9(1)(1999), 57-62.

[8] M. S. Ying, A New Approach for Fuzzy Topology (I), Fuzzy Sets and Systems 39(1991), 303-321.

[9] P. K. Lim, B. K. Ryou and K. Hur, Ordinary Smooth Topological Spaces, IJFIS. 12(1)(2012), 66-76.

[10] M. S. Cheong, G. B. Chae, K. Hur and S. M. Kim, The Lattice of Ordinary Smooth Topologies, Honam Math. J. 33(4)(2011), 453-465.

[11] M. Ying, A New Approach for Fuzzy Topology (III), Fuzzy Sets and Systems 55(1933), 195-207.

\section{저 자 소 개}

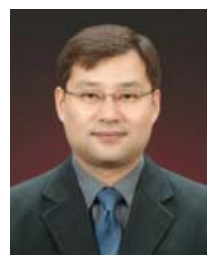

이정곤(Jeong Gon Lee)

Assistant Professor in Wonkwang University

관심분야: Measure Theory, Operator Theory, Category Theory, Hyperspace, Topology, Mathematics Education.

Phone : +82-63-850-6186

E-mail : jukolee@wku.ac.kr

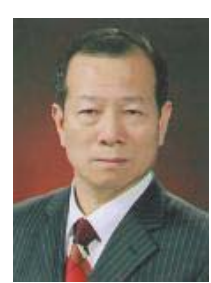

임평기( Pyung Ki Lim)

Professor in Wonkwang University

관심분야: Category Theory, Hyperspace, Topology.

Phone : +82-63-850-6559

E-mail : pklim@wonkwang.ac.kr

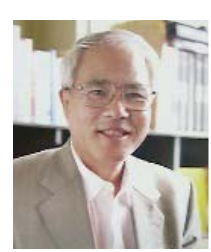

허 걸(Kul Hur)

Professor in Wonkwang University

관심분야: Category Theory, Hyperspace, Topology.

Phone : +82-63-850-6081

E-mail : kulhur@wonkwang.ac.kr 